\title{
Elemental Mapping of Prostate Tissue by Micro-SRIXE
}

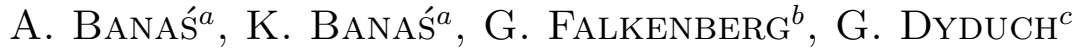 \\ AND W.M. KWIATEK ${ }^{a}$ \\ ${ }^{a}$ The Henryk Niewodniczański Institute of Nuclear Physics \\ Polish Academy of Sciences, Radzikowskiego 152, 31-342 Kraków, Poland \\ ${ }^{b}$ HASYLAB, DESY, Notkestr. 85, 22603 Hamburg, Germany \\ ${ }^{c}$ Dept. of Pathomorphology, Collegium Medicum, Jagiellonian University \\ Kopernika 7, 31-034 Kraków, Poland
}

\begin{abstract}
Synchrotron radiation-induced X-ray emission is now a proven analytical method for the determination of trace elements. Better insight of the role of trace elements in tissue samples can be gained by acquiring complete distribution maps of these elements. Our approach is based on the acquisition of digital maps. In this contribution we present two different methods for obtaining normalization matrix: first — based on argon intensity in the single spectrum and second - based on changes of beam current intensity. The region for the scan was carefully chosen according to the histological view of the sample. A total area was scanned with a step size of $15 \mu \mathrm{m}$ in each direction and measuring time of $30 \mathrm{~s}$ per pixel. The thickness of the samples was $15 \mu \mathrm{m}$. A polycapillary was used for beam focusing. At the energy of $18 \mathrm{keV}$ the beam size on the sample was approximately $15 \mu \mathrm{m}$ which is of the order of a cell diameter.
\end{abstract}

PACS numbers: 87.64.--t

\section{Introduction}

The spatial distribution of trace elements (TE) in tissues is involved in many biological functions of living organisms. Nowadays synchrotron radiation-induced $\mathrm{X}$-ray emission (SRIXE) is a proven analytical method for complete distribution maps of these elements $[1,2]$ according to minimum radiation damage to the sample, short time analysis, and low detection limit (below ppm level).

The crucial issue in data analysis is the method of normalization, because only in this way it is possible to obtain distribution maps of elements which are strictly connected with histological structures. Areas with high concentrations of some elements are later used in X-ray absorption near-edge structure (XANES) 
analysis which is useful to determine the oxidation state and the local environment of the absorbing atom(s). All the efforts that are made during the samples preparation, the data collection and the further qualitative processing of the data to increase the reliability of the final trace elements determination, may be negated by using wrong normalization.

In this contribution we present two different methods of normalization raw data based on:

- area under the argon's peak (AA) in the single spectrum,

- changes of the beam current during the scan (DC stand for values beam current monitor).

The importance of using accurate normalization procedures will be illustrated using data sets from the biological fields of application.

\section{Methods}

The measurements were carried out on the L-beam line at the HASYLAB, DESY (Germany). Synchrotron is characterized by the following parameters: electron energy $4.45 \mathrm{GeV}$, critical energy $16.04 \mathrm{keV}$, and maximum ring current approximately $120 \mathrm{~mA}$. During the experiments the polycapillary was used to obtain spectra from single points (beam size of $15 \mu \mathrm{m}$ in diameter was achieved). X-ray fluorescence was detected by use of a conventional HPGe X-ray detector positioned at $90^{\circ}$ to the incoming linear polarized X-ray beam and placed in the storage ring plane in order to minimize the intensity of the scattering X-rays. The energy resolution of the detector was $140 \mathrm{eV}$ for $5.9 \mathrm{keV}$. The angle between beam and sample and between sample and detector was fixed to $45^{\circ}$ each, in order to minimize the path length of X-rays in the sample.

All experiments were performed in air. The position of the sample, with respect to the X-ray beam, was changed by means of a computer controlled stepper motor allowing micrometric movements. Throughout the analysis the sample was supervised with a long distance zoom microscope with a magnification of 40 to $1200 \times$ and CCD-camera with a resolution of $3 \mu \mathrm{m}$. CCD camera was used for a visual control of the microbeam position on the sample. A Canberra 2060 Digital Signal Processor was used for collecting the spectra. The AXIL [3] software package was used for evaluation of the spectrum.

The adopted scan pattern was the same for all samples: from right to left for $x$-axis and from top to bottom for $y$-axis. The counting lifetime for each pixel (typical scans were about $450 \mu \mathrm{m} \times 450 \mu \mathrm{m}$ ) was $30 \mathrm{~s} /$ pixel (sufficiently long time to ensure good statistics) and the step size was $15 \mu \mathrm{m}$ in both directions. About 1000 spectra were typically collected during analysis. Measuring radiation emitted from each spot of samples was collected in ASCII format (files *.FIO). Information about position of scanned spot, beam current and time have been also stored within the ${ }^{*}$.FIO files. The peak areas of the fluorescence and scatter peaks for all 
spectra were fitted and displayed on-line in two-dimensional elemental maps automatically during the measurement using the AXIL and MICROXRF2 software. AXIL is a conventional program for spectrum analysis. The evaluation of energy dispersive X-ray spectra by means of AXIL was achieved by fitting the measured spectra with suited mathematical functions, using a non-linear least squares strategy. Special model (file *.INP) including information about Gaussian line shape centered around proper energy, lines positions, their relative amplitudes, distance between $K_{\alpha}$ and $K_{\beta}$ lines, and the shape of the background etc., was created prior to the actual spectrum analysis using X-ray library available in software. In the presented analysis the smooth filter (SF) background was chosen. SF procedure is based on the removal of rapidly varying structures in a spectrum by comparing the channel content $y_{i}$ with the channel content of its neighbours. For each spectrum the background was subtracted. All results of fitting procedures were saved in ASCII files with the extensions of *.ASR. Those files contain basically information about net peak areas, standard deviations for peak positions and chi-square for the fit of each peak.

The post processing procedure consists in:

- building the normalization matrices by extracting from single spectra information about calculated AA or DC (see introduction),

- rescaling of the results by using one of the normalization matrices (in this context rescaling means division of measured concentration matrix of each element by one of the normalization matrices),

- visualization of the normalized matrices with concentrations of trace elements by colour scale maps.

\section{Results and discussion}

Specimens for the analysis were taken from frozen prostate tissues obtained from patients with prostate cancer. The samples were cut into $15 \mu \mathrm{m}$ sections and mounted on $2.5 \mu \mathrm{m}$ Mylar film. Pre-studies of the samples were performed with optical microscopy by histologists in order to fully characterize the samples. From these studies certain areas were selected for further analysis. Figure 1 presents histological view of prostate tissue $450 \mu \mathrm{m} \times 450 \mu \mathrm{m}$ (a) and non-normalized $\mathrm{Cl}$ distribution (b).

Figure 2 presents plot of AA and DC versus time. Those data were extracted from single spectra in order to built two different normalization matrices for AA (Fig. 3a) and DC (Fig. 3b). As one can notice, changes of AA during scanning are much less stable as compared to beam current changes. These local fluctuations of AA may affect final concentrations of elements distributions and uncontrolled losses of important information and wrong choice of appropriate spot for further XANES analysis which is presented in Fig. 3 ((c) and (d)). 



Fig. 1. (a) Histological view of prostate tissue, (b) map of non-normalized $\mathrm{Cl}$ distribution.

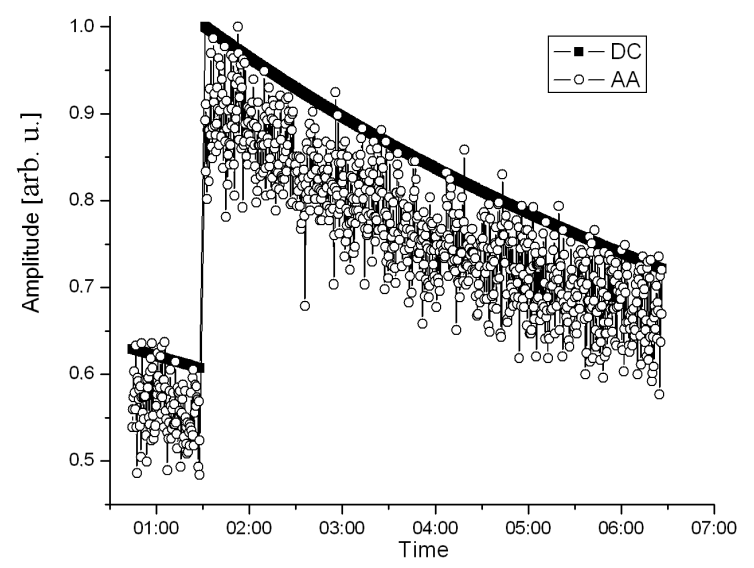

Fig. 2. Plot of area under argon's peak and beam current versus time.

\section{Conclusions}

In biological applications the maps may give a direct and clear observation of element occurrences in different regions of the sample. The proper distributions of the certain elements are strictly required in XANES analysis.

In this paper we introduced a technique of data normalization based on changes of the beam current during the experiment. In comparison with the standard method which is based on area under the argon's peak and which is commonly used by the others [4], our approach gives much more reliable results. Using matrices composed of argon concentration in normalization procedures provides artefacts in final distribution maps.

The feasibility of the new method has been demonstrated by measurements of the biological sample. These results demonstrate that SRIXE technique can be 


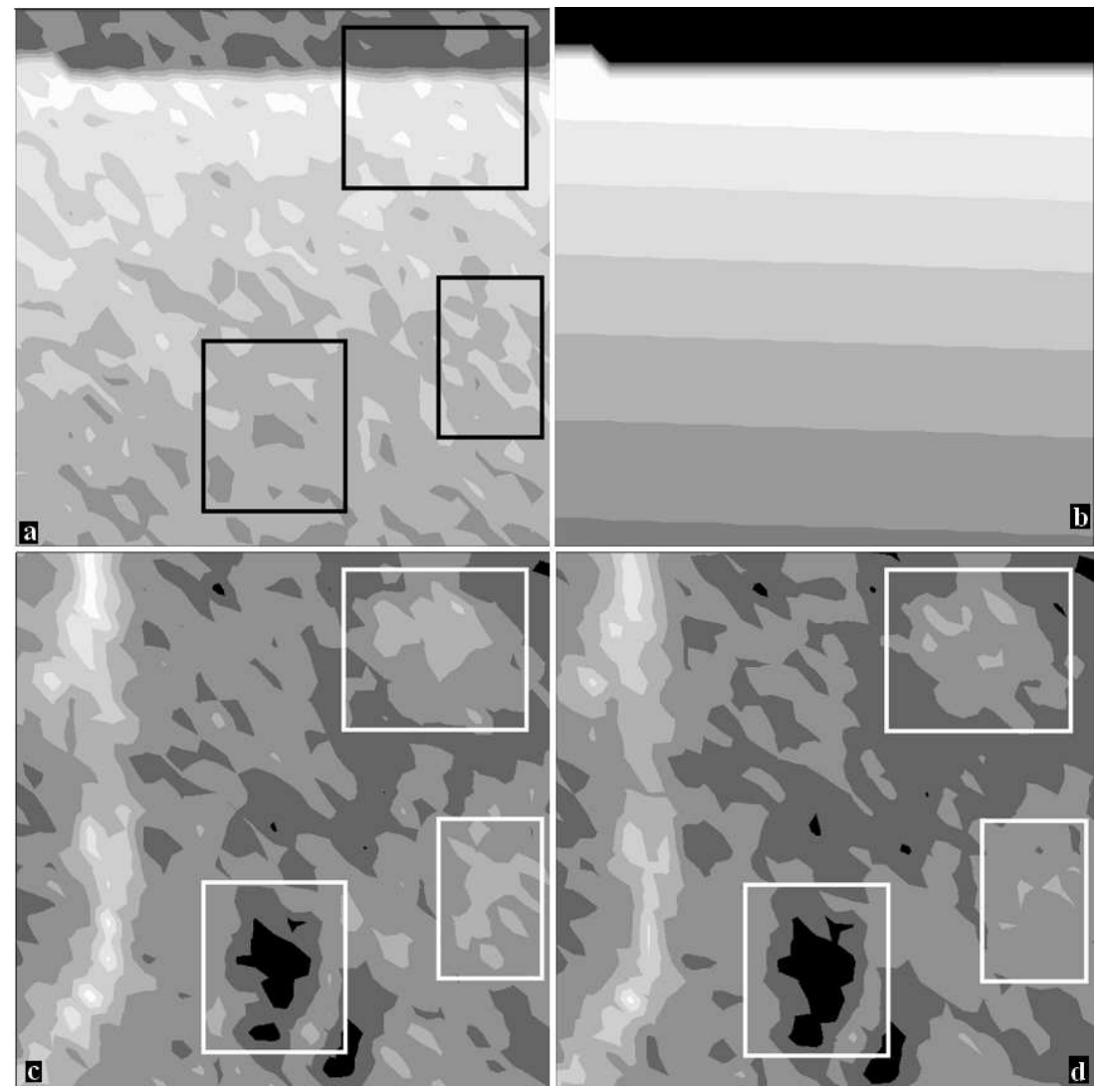

Fig. 3. Maps: visualization of normalization matrices for: (a) area under argon's peak (AA), (b) beam current (DC), (c) AA, (d) Cl distribution normalized by DC. Regions of interest indicate differences in final $\mathrm{Cl}$ concentration due to various normalization procedures.

successfully used for spatial elemental distribution determination as well as local elemental concentration levels in human tissues.

Elemental maps which depict the local concentration of a certain element have great potential for use in various kinds of biomedical research. SRIXE is a well-established technique for determining local elemental concentrations, because of its low detection limit of only a few $\mu \mathrm{g} / \mathrm{g}$ and its high spatial resolution. In many of the applied studies conducted in these fields, normalization reliability is an important issue.

\section{Acknowledgments}

This work has been supported by HASYLAB, DESY, Hamburg, Germany under project II-02-010, the IHP-Contract HPRI-CT-1999-00040/2001-00140 of the European Commission. 


\section{References}

[1] W.M. Kwiatek, A. Banas, K. Banas, M. Gajda, M. Gałka, G. Falkenberg, T. Cichocki, J. Alloys Comp., in press.

[2] K.W. Jones, W.J. Berry, D.J. Borsay, H.T. Cline, W.C. Conner Jr, C.S. Fullmer, X-Ray Spectrom. 26, 350 (1999).

[3] P. Van Espen, K. Janssens, I. Swensters, AXIL X-Ray Analysis Software - Users Manual, Canberra-Packard, Benelux 1989, p. 48.

[4] J. Chwiej, M. Szczerbowska-Boruchowska, M. Lankosz, D. Adamek, J. Alloys Comp., in press. 